OPEN ACCESS

Edited by:

Anna M. Borghi,

Sapienza Università di Roma, Italy

Reviewed by:

Stephen V. Shepherd, The Rockefeller University,

United States

Claudia Gianelli,

Universität Potsdam, Germany

*Correspondence:

Luisa Sartor

luisa.sartori@unipd.it

Specialty section: This article was submitted to

Cognition,

a section of the journal

Frontiers in Psychology

Received: 07 March 2018

Accepted: 19 July 2018

Published: 09 August 2018

Citation:

Betti S, Zani G, Granziol U, Guerra S,

Castiello U and Sartori L (2018) Look

at Me: Early Gaze Engagement

Enhances Corticospinal Excitability

During Action Observation.

Front. Psychol. 9:1408.

doi: 10.3389/fpsyg.2018.01408

\section{Look at Me: Early Gaze Engagement Enhances Corticospinal Excitability During Action Observation}

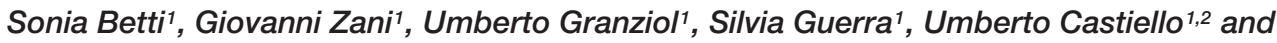 \\ Luisa Sartori1,3*
}

'Dipartimento di Psicologia Generale, Università di Padova, Padova, Italy, ${ }^{2}$ Centro Beniamino Segre, Accademia Nazionale dei Lincei, Rome, Italy, ${ }^{3}$ Center for Cognitive Neuroscience, Università di Padova, Padova, Italy

Direct gaze is a powerful social cue able to capture the onlooker's attention. Beside gaze, head and limb movements as well can provide relevant sources of information for social interaction. This study investigated the joint role of direct gaze and hand gestures on onlookers corticospinal excitability (CE). In two experiments we manipulated the temporal and spatial aspects of observed gaze and hand behavior to assess their role in affecting motor preparation. To do this, transcranial magnetic stimulation (TMS) on the primary motor cortex (M1) coupled with electromyography (EMG) recording was used in two experiments. In the crucial manipulation, we showed to participants four video clips of an actor who initially displayed eye contact while starting a social request gesture, and then completed the action while directing his gaze toward a salient object for the interaction. This way, the observed gaze potentially expressed the intention to interact. Eye tracking data confirmed that gaze manipulation was effective in drawing observers' attention to the actor's hand gesture. In the attempt to reveal possible timelocked modulations, we tracked $\mathrm{CE}$ at the onset and offset of the request gesture. Neurophysiological results showed an early CE modulation when the actor was about to start the request gesture looking straight to the participants, compared to when his gaze was averted from the gesture. This effect was time-locked to the kinematics of the actor's arm movement. Overall, data from the two experiments seem to indicate that the joint contribution of direct gaze and precocious kinematic information, gained while a request gesture is on the verge of beginning, increases the subjective experience of involvement and allows observers to prepare for an appropriate social interaction. On the contrary, the separation of gaze cues and body kinematics can have adverse effects on social motor preparation. CE is highly susceptible to biological cues, such as averted gaze, which is able to automatically capture and divert observer's attention. This point to the existence of heuristics based on early action and gaze cues that would allow observers to interact appropriately.

Keywords: action observation, gaze, attention, TMS, complementary actions

\section{INTRODUCTION}

In humans, eye contact may signal an approaching intention from the gazer toward the perceiver, and it is critical for communication and social interactions (Kleinke, 1986; Senju and Johnson, 2009; Schilbach et al., 2013; Hamilton, 2016). An early sensitivity to noticing people's eye region and gaze direction is already detectable in newborns, which prefer faces showing a direct gaze 
compared to faces with averted or closed eyes (Batki et al., 2000; Farroni et al., 2002). Eye contact is so relevant in social development that a failure to develop typical gaze behavior is one of the earliest signals of social disorders, such as autism (BaronCohen, 1995a; Hamilton, 2016). Since the ability to rapidly detect other's gaze represent an advantage for the human species, a peculiar eye configuration together with dedicated mechanisms for gaze processing have evolved to allow for an easy detection of gaze direction (Kobayashi and Kohshima, 2001). In line with this, Baron-Cohen (1995b) hypothesized the existence of an eyedirection detector (EDD) in humans, specialized in computing eye-gaze direction. In social contexts, an agent's gaze can provide a cue possibly influencing the orientation of attention in given portions of space (for reviews, see Emery, 2000; Langton et al., 2000; Frischen et al., 2007). Observing another's averted gaze can trigger in the onlooker a rapid and automatic shift of attention toward the gazed direction (e.g., Driver et al., 1999; Langton and Bruce, 1999; Friesen et al., 2005).

Together with eye-gaze, also head and limbs movements may determine a shift of attention toward specific aspects of the environment. Prinsen et al. (2017) showed their participants video clips of a hand opening and closing in front of a face gazing toward or away with respect to the observers. Direct eye contact, compared to averted gaze presentation, enhanced direct matching in the observers' hand muscles (Prinsen et al., 2017). Other studies specifically explored the relationship between grasping actions, gaze and attention (see Atkinson et al., 2017 for an extensive review), addressing how observing another's gaze and grasping behavior influences our own actions (e.g., Castiello, 2003; Pierno et al., 2006; Letesson et al., 2015). For example, Letesson et al. (2015) presented participants video clips in which an actor looked at the camera, then directed his gaze toward one of two objects in front of him and grasped it. Participants were then requested to perform a reach-to-grasp action toward target objects of the same or different size compared to the one grasped by the actor. Gaze and action cues differently modulated speed and accuracy toward the target object, but the combined availability of both cues led to a more accurate action execution. The relation between an agent's hand posture and gaze in a reach to grasp task has also been considered in the context of complementary actions. Complementary actions (from Latin complementum; i.e., that fills up) are a specific class of movements which differ, while interacting, with observed ones (Sebanz et al., 2006; Sartori and Betti, 2015; Sartori, 2016). In a study by Innocenti et al. (2012), participants were requested to reach and lift a bottle in the presence of an empty glass, while their movement kinematics was recorded. When a conspecific produced a complementary request gesture (i.e., holding the empty glass while displaying a direct gaze to the participant), their lifting action was significantly interfered by the newly activated motor program of pouring, so that the grasping action on the bottle was slowed down (Innocenti et al., 2012).

Here we capitalize on previous research on complementary actions probing the motor system by means of transcranial magnetic stimulation (TMS) coupled with electromyography (EMG) (for reviews see Sartori and Betti, 2015; Sartori, 2016). Seeing an actor in a frontal position with an open hand signaling a request near a salient object, strategically placed close to the participant, induces a modulation in the observer's muscular activity that is consistent with the intention to accept the request (i.e., grasping the object) rather than with the tendency to resonate with the observed action. Complementary response preparation seems to be very prompt and time-locked to the kinematics of other's movement. The functional shift, in particular, indicates the ability to untie the automatic tendency to mirror another's actions in order to prepare a complementary response (Sartori et al., 2013). Notably, this effect is founded on the "readiness to interact" (i.e., the willingness to engage in socially meaningful situations; Di Paolo and De Jaegher, 2012), since an arrow cue pointing toward the object instead of the hand gesture does not produce the same motor activations (Sartori et al., 2011b). Along these lines, Betti et al. (2017) recently manipulated participant's attention while they were presented with action sequences requiring (or not) an interactive response. In particular, videos of an actress grasping a spoon full of sugar, pouring some sugar into a mug next to her on a table and then stretching toward a mug out of her reach but close to the participant - as to pour some left sugar in it, were presented. Diverting attention by means of an exogenous cue (i.e., a red dot) interfered with the mirroring of noninteractive actions, but did not affect complementary response preparation. Here we extended previous evidence by adopting a more ecological setting: we manipulated the shift of other's gaze in a social context eliciting complementary responses. In a preliminary study (Experiment 1) we investigated whether observing a request gesture while the actor directs his gaze toward a salient target object or toward the observer (i.e., direct gaze) differently affects his/her corticospinal excitability (CE). We reasoned that if direct gaze increases the observer's social engagement, then $\mathrm{CE}$ in the corresponding muscle should grow. On the contrary, if motor preparation increases when the observed gaze points to the object, this might suggest that object salience and action predictability are the crucial nodes of this effect. The correlation of this neurophysiological index (CE) with the subjective experience of involvement was also investigated. In Experiment 2 we slightly modified the paradigm. Participants observed the actor looking at them (direct gaze) and then directing his gaze to the salient object or away from it. In real life situations, gaze cues and body kinematics are critical in guiding an observer's behavior in a context-dependent manner and their separation, as stressed by Reader and Holmes (2016), may have adverse effect on the validity of social interaction experiments. In Experiment 2, the combined manipulation of gaze and body cues, and the adoption of different TMS timings were intended to better unveil the mechanisms underlying social motor preparation.

\section{EXPERIMENT 1}

In Experiment 1 we assessed CE modulations while participants observed an actor performing a request gesture while directing his gaze toward a salient target object or toward the observer (direct gaze). In order to correlate this neurophysiological 
index of motor preparation with the subjective experience of involvement, at the end of the session participants fulfilled a questionnaire to quantify their willingness to interact.

\section{Materials and Methods}

\section{Ethics Statement}

The experiments were approved by the ethics committee of the University of Padua $\left(\mathrm{N}^{\circ} 1817\right)$, in accordance with the Declaration of Helsinki (Sixth revision, 2008). All participants gave their written informed consent and were financially compensated for their participation.

\section{Participants}

Thirty-three naïve volunteers (18 female and 15 male, aged between 21 and 30 years, mean age 23.6 years) took part in the experiment. All participants were right-handed, as assessed with the Edinburgh Handedness Inventory (Oldfield, 1971), with normal or corrected-to-normal visual acuity. They were all screened for TMS exclusion criteria and for neurological, psychiatric and medical problems (Wassermann, 1998; Rossi et al., 2009). A right-handed non-professional actor (male, 29 years old) was recruited for video-clips recording.

\section{Experimental Stimuli}

Two video clips were adopted as experimental stimuli:

(a) Object gaze: the actor grasped a spoon full of sugar with a precision grip (PG; i.e., the opposition of the thumb to the index finger). Then he poured half sugar into a mug placed next to him and he finally stretched out his arm toward a mug out of his reach, with some sugar left in the spoon. The mug was strategically placed near the observer, in the right corner of the screen, thus affording a whole hand grasp (WHG; i.e., the opposition of the fingers to the palm) to be handled. The actor was instructed to follow his hand movements in a natural way with his gaze and to look at the mug while his arm was stretching toward it (Figure 1A).

(b) Direct gaze: the actor performed the same action as in the "Object gaze" condition, but at the end of the social request gesture he lifted his head and gazed toward the observer with his arm stretched out (Figure 1B).

Note that this social request would require the observer's intervention to lift the mug and to complete the complementary action. This way, the observed movement (i.e., PG) was specifically mismatched with the one required to interact in a complementary fashion (i.e., WHG). Each video lasted $6210 \mathrm{~ms}$ and the animation effect was obtained by presenting a series of single frames each lasting $30 \mathrm{~ms}$ (resolution $1920 \times 1080$ pixels, color depth 32 bits) following the first frame lasting $500 \mathrm{~ms}$.

\section{Procedure}

Participants were tested individually in a single experimental session lasting approximately $1 \mathrm{~h}$ and a half. They were seated in a comfortable armchair with the right arm positioned on a pillow and the head on a fixed head rest. They were instructed to remain as still and relaxed as possible while watching the video clips that were presented on a 24 " monitor (resolution $1920 \times 1080$ pixels, refresh frequency $120 \mathrm{~Hz}$ ) set at eye level (the eye-screen distance was $80 \mathrm{~cm}$ ). No specific task was given to participants; however, they were told that at the end of the experiment they would be questioned about the stimuli presented (i.e., post-experiment questionnaire) to ensure attention to the video clips. TMSinduced motor-evoked potentials (MEPs) were acquired from the participants' right first dorsal interosseous (FDI) and abductor digiti minimi (ADM) muscles of the right hand. A single TMS pulse was released during each video presentation at the end of the action sequence (5090 ms), namely when the actor's arm was

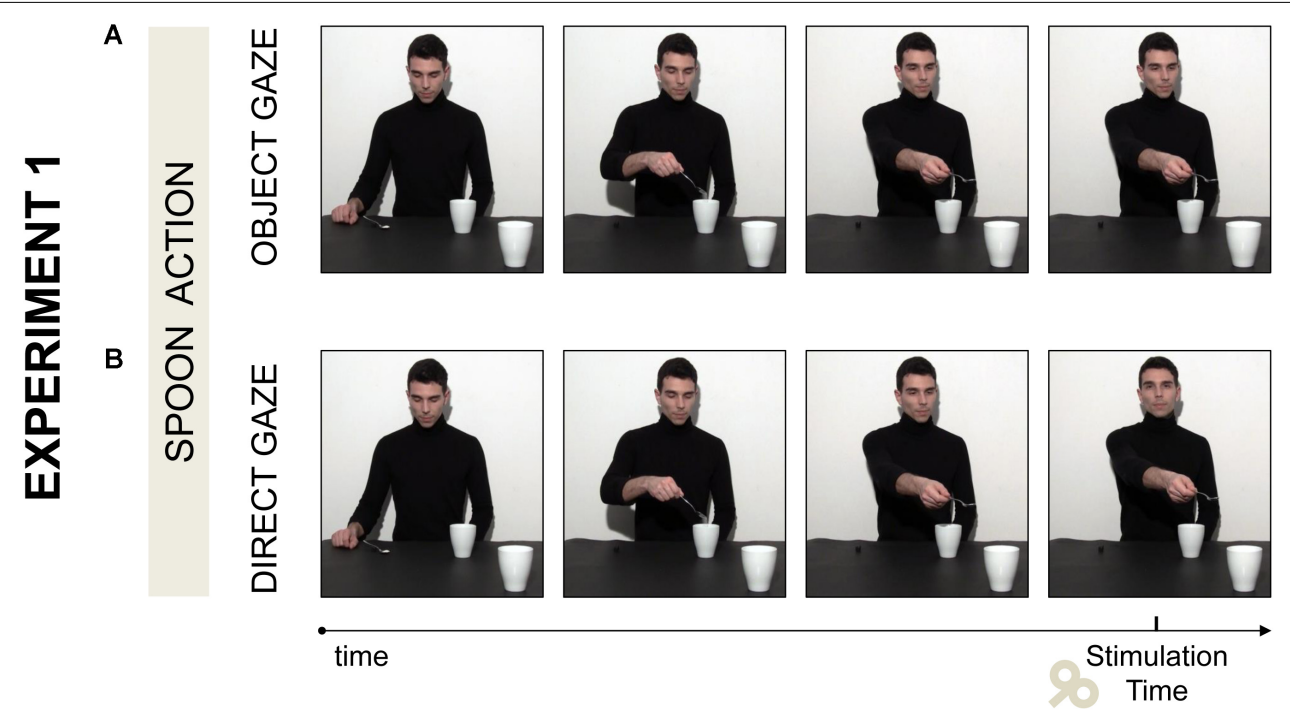

FIGURE 1 | Two video clips were adopted in Experiment 1. (A) In the Object gaze video the actor stretched out his arm toward a mug out of his reach and looked at it. (B) In the Direct Gaze video the actor performed the same action, but at the end of the social request gesture he lifted his head and gazed toward the observer while his arm was stretched out. Single pulse TMS was delivered at the offset of the social request gesture. 
already stretched out toward the out-of-reach mug and his gaze was directed to the mug (Object gaze condition; Figure 1A) or to the observer (Direct Gaze condition; Figure 1B). The order of the video clips was randomized across participants. A total of 60 MEPs ( 2 muscles $\times 2$ conditions $\times 15$ repetitions) were recorded for each participant. Prior and after the experimental block, each participant's baseline CE was assessed by acquiring 15 MEPs while they passively watched a white fixation cross on a black background presented on the computer screen. The average MEP amplitudes recorded during the two baseline periods (30 MEPs in total) was used to set each participant's individual baseline for data normalization procedures. An inter-pulse interval lasting $10 \mathrm{~s}$ was adopted between trials in order to avoid any short-term conditioning effect (Classen et al., 2000). During this interval participants were reminded to remain fully relaxed for $5 \mathrm{~s}$ and a fixation cross was presented for the remaining $5 \mathrm{~s}$. The presentation of a fixation cross before each trial ensured that participants always started the trial by observing the videos from a neutral gaze position. Stimuli presentation, timing of TMS stimulation and EMG recordings were managed by E-Prime V2.0 software (Psychology Software Tools, Inc., Pittsburgh, PA, United States) running on a computer.

\section{TMS and EMG recording}

Single-pulse TMS was administered using a $70 \mathrm{~mm}$ figure-ofeight coil connected to a Magstim BiStim2 stimulator (Magstim Co., Whitland, United Kingdom). Pulses were delivered to the hand region of the left primary motor cortex (M1). The coil was placed tangentially to the scalp, with the handle pointing laterally and caudally (Basil-Neto et al., 1992; Mills et al., 1992), in correspondence with the optimal scalp position (OSP) where MEPs with maximal amplitude were recorded simultaneously from the FDI and ADM muscles with the minimum stimulation intensity. To find the individual OSP, the coil was moved in steps of $0.5 \mathrm{~cm}$ until the position was reached. Once the OSP was found, it was marked on a tight-fitting cap worn by the participant. Then, the individual resting motor threshold (rMT), that is the lowest stimulus intensity at which TMS is able to generate MEPs of at least $50 \mu \mathrm{V}$ in relaxed muscles in 5 out of 10 consecutive pulses (Rossini et al., 1994), was determined for the less excitable muscle (ADM). The stimulation intensity was then set at $120 \%$ of the rMT to record a clear and stable MEP signal throughout the experiment. rMT ranged from 32 to $56 \%$ ( mean $=40 \%$ and SD $=5.1$ ) of the maximum stimulator output. During the experimental sessions the coil was held by a tripod and continuously checked by the experimenters to maintain a constant positioning with respect to the marked OSP. MEPs were recorded simultaneously from the FDI and ADM muscles of the participant's right hand. These muscles were chosen because of their differential activation during the observation of PG and WHG (e.g., Betti et al., 2018). In particular, ADM is only activated for WHG, whereas FDI is modulated during observation of both types of grasp (e.g., Gangitano et al., 2001). This aspect is crucial for the present manipulation, since we expect a muscular-specific activation for the ADM muscle when a request is made toward the large mug (i.e., a WHG) but not toward the small coffee cup, whereas the control muscle (FDI) should be activated in both cases. The EMG signal was recorded by means of two pairs of surface $\mathrm{Ag} / \mathrm{AgCl}$ electrodes (1 $\mathrm{cm}$ diameter) placed in a bellytendon montage, with the active electrode placed over the muscle belly and the reference over the interphalangeal joint. The ground electrode was positioned over the participant's left wrist. Skin impedance, evaluated at rest prior to beginning the experimental session, was considered of good quality when below the threshold level (5 Ohm). Electrodes were connected to an isolable portable ExG input box linked to the main EMG amplifier for signal transmission via a twin fiber optic cable (Professional BrainAmp ExG MR, Munich, Germany). The raw myographic signals were band-pass filtered $(10 \mathrm{~Hz}-1 \mathrm{kHz})$, amplified prior to being digitalized (5 $\mathrm{kHz}$ sampling rate), and stored on a computer for off-line analysis. EMG data were collected for $300 \mathrm{~ms}$ after the TMS pulse by using Brain Vision Recorder software (Brain Products GmbH, Munich, Germany).

\section{Post-experimental questionnaire}

At the end of the experimental session participants were instructed to express on a five-point Likert scale (ranging from "Not at all" to "Very much") their agreement or disagreement with four statements for each condition. The order of the conditions which the sentences referred to was counterbalanced between participants. Hereafter the adopted items (translated from Italian): (Q1) "I felt involved in the action"; (Q2) "At the end of the video I would have grabbed the nearest mug"; (Q3) "At the end of the video, I had the impression that the boy wanted to interact with me"; and (Q4) "At the end of the video I wanted to join the action". These sentences were adopted to assess the experimental stimuli were effective in modulating perceived engagement and to quantify the subjective experience of involvement experienced by each participant during the experiment. One of the hallmark of our study was to explore the relationship between the participants' perceived level of engagement and the corresponding CE modulations during video observation. To do so, we decided to cluster our sample in two groups of responders, in order to examine whether the different attitude toward the observed scenes (i.e., low and high engagement) was associated with different patterns of motor activations.

\section{Data Analysis}

Data were analyzed offline using Brain Vision Analyzer software (Brain Products GmbH, Munich, Germany) for EMG data and the softwares R (version 3.3.2; R Core Team, 2016) for statistical analysis. The MEP peak-to-peak amplitude $(\mathrm{mV})$ for FDI and ADM muscles was determined as a measure of participants' CE. Trials in which any EMG activity greater than $100 \mu \mathrm{V}$ was present in the $100 \mathrm{~ms}$ window preceding the TMS pulse were discarded to prevent contamination of MEP measurements by background EMG activity. Trials contaminated by muscular pre-activation and values exceeding the $3 \mathrm{SD}$ from the mean were excluded as outliers $(<5 \%)$. Ratios were computed using the participant's individual mean MEP amplitude recorded during the two baseline periods (MEP ratio = MEPobtained $/ \mathrm{MEPb}$ aseline). Statistical analyses of the data were performed using a linear mixed-effects model on MEPs. Two predictors were used as 
fixed effects of the model, namely the Muscle (ADM and FDI) and Gaze direction (Object gaze and Direct gaze) predictors. The interaction between predictors was inserted in the model. Participants were set as a random factor (random intercept model), in order to assess the individual variability. The mixed models were performed using the $\mathrm{R}$ packages lme4 (Bates et al., 2014). The significance of the fixed effects was tested by means of a Wald Chi-Square test, performed by using the $\mathrm{R}$ package Car (Fox and Weisberg, 2011). A significance threshold of $p<0.05$ was set for all statistical analysis. Each time a statistically significant effect was found, multiple comparisons were performed using the lsmeans package (Lenth, 2016). The degrees of freedom of such comparisons were computed using the Satterthwaite method, while the $p$-values were adjusted by means of the Tukey method (Gaylor and Hopper, 1969; Lenth, 2016). A mixed effects model was tested on the questionnaire scorings, with Condition (Object Gaze and Direct Gaze) and Item (Q1, Q2, Q3, and Q4) set as fixed effects, and subjects as random effect. Finally, to explore a possible link between the perceived engagement with the observed action and the observer's CE, Pearson's correlations between questionnaire scorings and MEP values were computed for each experimental condition and muscle. Bonferroni's correction for multiple comparisons was applied to prevent Type- 1 errors. Moreover, a cluster analysis was performed on the post-experimental questions running a $\mathrm{k}$-means analysis. Two centroids for the final cluster membership configuration were set. The choice of selecting two centroids (groups) was taken in order to create two theoretical groups of responders: High and Low responders. These two groups were then used as a between factor for simple t tests to compare MEP scores between groups.

\section{Results and Discussion}

The results of the mixed model on the questionnaire responses confirmed that adopted stimuli were effective in modulating perceived engagement. A main effect of Condition $\left(\chi^{2}(1)=45.81\right.$, $p<0.001)$, Item $\left(\chi_{(3)}^{2}=27.72, p<0.001\right)$ and a significant interaction between Condition and Item $\left(\chi^{2}(3)=13.48, p<0.01\right)$ were found (see also Supplementary Table 1). In particular, Direct Gaze compared to Object Gaze conditions increased the scoring for Items Q1 ("I felt involved in the action"; $t_{(224)}=-4.51, p<0.001$ ) and Q3 ("At the end of the video, I had the impression that the boy wanted to interact with me"; $\left.t_{(224)}=-5.78, p<0.001\right)$. No statistically significant effects on MEP data were found $\left(p_{s}>0.05\right.$; see also Supplementary Table 2). Nonetheless, a positive correlation emerged for the Direct Gaze condition between the reported tendency to grab the nearest mug at the end of the observed action (Q2) and MEPs in the corresponding ADM muscle $\left(r_{(31)}=0.479\right.$, $p=0.020$; Figure 2A). Indeed, when considering the two clusters of responders (see Figure 2C), we found higher ADM MEP normalized scores for the High Responders than for the Low responders in the Direct Gaze condition $\left(t_{(27.35)}=2.54, p=0.017\right.$; Figure 2B). The more the participants felt involved in the action, the higher was the activation in the muscle required for the interaction. The presence of a consistent subgroup of Low Responders might have affected the neurophysiological effect on
MEPs. Therefore we decided to deeply consider this issue in Experiment 2.

\section{EXPERIMENT 2}

Experiment 1 was not effective in eliciting CE modulation. This was possibly due to some alternative explanations (or to their combination): (i) participants were not socially sensitive; (ii) gaze manipulation was ineffective; and (iii) TMS stimulation timing was inappropriate. In order to disentangle these variables, a new Experiment was conceived. First of all, the Reading the Mind in The Eyes Test (RMET), a well-recognized test of social sensitivity (Baron-Cohen et al., 2001) was administered to participants. Then, a new paradigm was adopted in order to better control the gaze manipulation: after pouring in the close mug/cup (Step A), the actor started his social request gesture (Step B) while displaying direct eye contact, and then he looked at the salient object. In two control conditions, the actor's gaze was diverted from the action and directed toward a neutral part of the visual scene. This way, direct and averted gaze potentially expressed the request to interact or not, respectively (George and Conty, 2008; Senju and Johnson, 2009). Moreover, eye-tracking recordings were acquired during observation of all the action sequences to validate that our manipulation had an effect on observers' gaze behavior. Lastly, in the attempt to reveal possible time-locked modulations, we thoroughly investigated Step B by tracking participants' $\mathrm{CE}$ at both the onset $\left(\mathrm{T}_{1}\right)$ and offset $\left(\mathrm{T}_{2}\right)$ of the request gesture. As a further control, we introduced a double dissociation to test the muscle-specificity of the neurophysiological effect. Two different types of grasps were shown in the videos (i.e., a precision grip on a spoon or a power grip on a thermos) to elicit two different complementary motor responses in the observer (i.e., a power grip on a mug or a precision grip on a coffee cup). Since different types of grasp differently involve the examined muscles (i.e., FDI and ADM), we hypothesized that only the target object affording a WHG (i.e., the mug) would have specifically activated ADM muscle. Whereas the control muscle (FDI) should be activated in both types of grasp.

\section{Materials and Methods Participants}

Thirty-three individuals were recruited. The data from three participants could not be used in the analysis due to technical problems. Therefore, thirty participants (20 female and 10 male, aged between 19 and 29 years, mean 23 years) with the same characteristics of those who took part in Experiment 1 were included in the analysis.

\section{Experimental Stimuli}

Four video clips were adopted as experimental stimuli:

(a) Spoon-Engaged: an actor grasped a spoon full of sugar and poured some sugar into a mug placed next to him (Step A). Then he started stretching out his arm toward a mug out of his reach (Step B) while directing his gaze toward the 

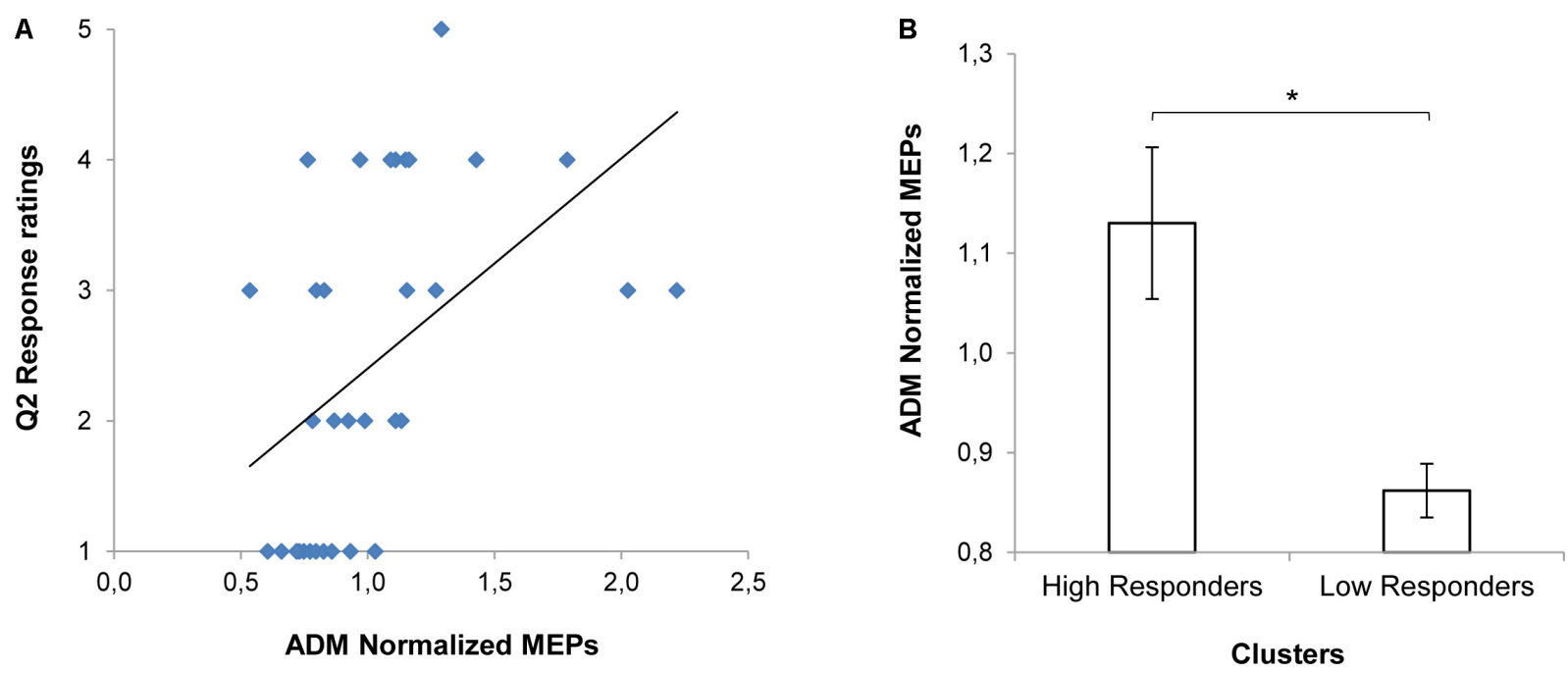

C

\section{Question $1 \quad$ Question $2 \quad$ Question $3 \quad$ Question 4}

\begin{tabular}{lllll}
\hline High Responders & 4.14 & 3.10 & 4.43 & 3.62 \\
\hline Low Responders & 2.42 & 1.33 & 2.00 & 1.33 \\
\hline
\end{tabular}

FIGURE 2 | MEP values and Questionnaire scorings for Experiment 1. A positive correlation between the reported tendency to grab the nearest mug at the end of the observed action (Q2) and ADM MEP values for the Direct Gaze condition is shown in panel (A). ADM MEP values for the High and the Low Responders in the Direct Gaze condition are graphically represented in panel (B). Asterisk indicates a statistically significant difference $(p<0.05)$. Bars refer to the standard errors. Mean scorings for each item of the Questionnaire on the basis of the clusters (High and Low responders) are reported in panel (C).

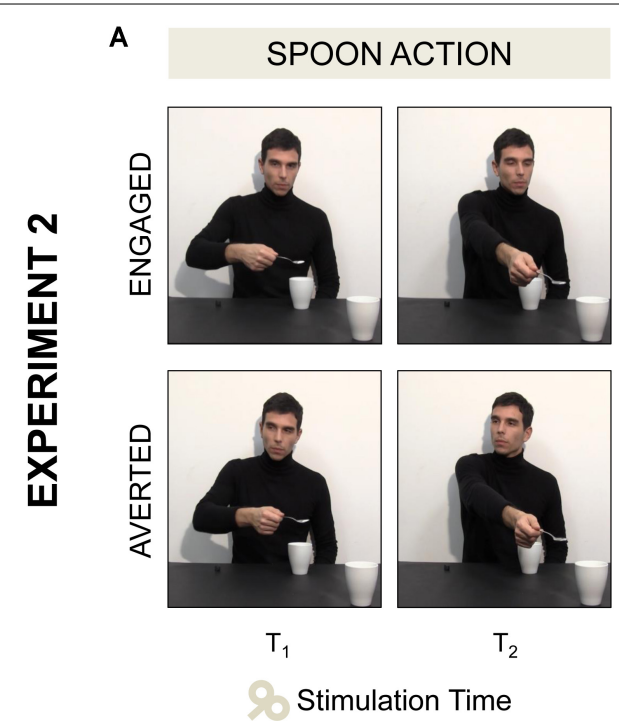

B

THERMOS ACTION

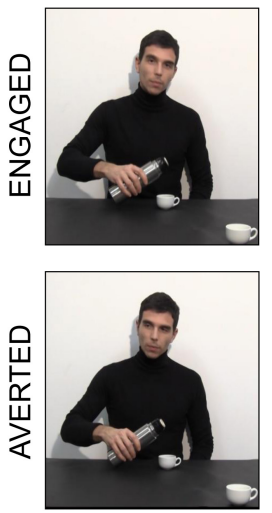

$\mathrm{T}_{1}$
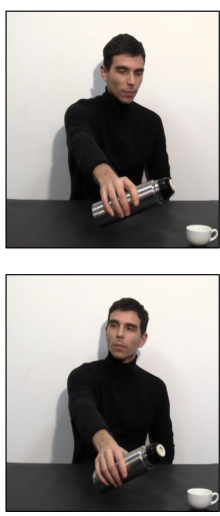

$\mathrm{T}_{2}$

FIGURE 3 | Four video clips were adopted in Experiment 2. (A) In the Spoon-Engaged video the actor started stretching out his arm toward a mug out of his reach while directing his gaze toward the observer ( $T_{1}$, see the first frame), then he completed the request gesture while gazing at the mug $\left(T_{2}\right.$, see the second frame).

(A) In the Spoon-Averted video the actor performed the very same "Spoon" action, but when stretching out his arm toward the out-of-reach mug he turned away his gaze pointing to his right. (B) In the Thermos-Engaged and Thermos-Averted videos the same action sequences were shown, except for the type of grasp (WHG) and the target object (coffee cup). 
observer $\left(T_{1}\right)$ and he completed the request gesture while gazing at the mug $\left(\mathrm{T}_{2}\right.$; Figure $\left.3 \mathrm{~A}\right)$.

(b) Spoon-Averted: the actor performed the very same "Spoon" gesture throughout the video, but during Step B he started turning his gaze to his right $\left(\mathrm{T}_{1}\right)$ and he completed the request gesture while gazing away $\left(\mathrm{T}_{2}\right.$; Figure $\left.3 \mathbf{A}\right)$

(c) Thermos-Engaged: the actor grasped a thermos and poured some coffee into a coffee cup placed next to him on a table (Step A). Then he started stretching out his arm toward a cup out of his reach while directing his gaze toward the observer $\left(T_{1}\right)$ and he completed the request gesture while gazing at the cup $\left(\mathrm{T}_{2}\right.$; Figure 3B).

(d) Thermos-Averted: the actor performed the very same "Thermos" gesture throughout the video, but during Step $\mathrm{B}$ he started turning his gaze to his right $\left(\mathrm{T}_{1}\right)$ and he completed the request gesture while gazing away $\left(\mathrm{T}_{2}\right.$; Figure 3B).

We specifically manipulated observers' shifts of attention and social involvement by means of actor's eye-gaze and head direction. The actor was instructed to keep looking at his hand until he had finished pouring (Step A) and then to unfold the social request action while performing either an interactive or an averted gaze (Step B). Notably, the type of grasp observed and the one required to respond to the social request were reciprocally mismatched in all the experimental conditions. All the videos underwent a post-hoc kinematic analysis using a digitalization technique (VideoTrack; Ab.Acus, Milano, Italy). Each movement was tracked by manually assigning a marker to the model's wrist, nose and eyes. This analysis allowed excluding that any difference in terms of hand gesture was present across Engaged/Averted conditions, despite gaze and head movements differed. Notably, this procedure also allowed checking that gaze and head movements were always convergent. Each video lasted $9680 \mathrm{~ms}$ and the animation effect was obtained by presenting a series of single frames each lasting $40 \mathrm{~ms}$ (resolution $1920 \times 1080$ pixels, color depth 32 bits) following the first frame lasting $500 \mathrm{~ms}$.

\section{Procedure}

Transcranial magnetic stimulation-induced MEP from the right $\mathrm{ADM}$ and FDI muscles were acquired during video observation. Eye-tracking measures were used to investigate overt attention allocation during the observation of the videos presented in the TMS session. Lastly, participants completed the Italian version of the RMET to evaluate their ability to attribute complex mental states from eyes' observation.

\section{Transcranial magnetic stimulation and electromyographic recording}

The TMS-EMG procedure in Experiment 2 was identical to Experiment 1, with the following exceptions regarding the stimulation timing and the type of videos. TMS-induced MEP from the right $\mathrm{ADM}$ and the right FDI muscles were acquired once for each video presentation at one of two possible time points: $\left(T_{1}\right)$ when the actor's wrist trajectory started to move toward the cup/mug next to the observer (onset of Step B; $5940 \mathrm{~ms}) ;\left(\mathrm{T}_{2}\right)$ when the actor's arm was completely stretched toward the cup/mug near to the observer (offset of Step B; $7500 \mathrm{~ms}$; see Figure 3B). Ten MEPs were acquired for each muscle (ADM and FDI), experimental condition (Spoon and Thermos), Gaze direction (Engaged and Averted) and Timing of stimulation $\left(\mathrm{T}_{1}\right.$ and $\left.\mathrm{T}_{2}\right)$, resulting in a total of 160 MEP per participant $(2$ muscles $\times 10$ repetitions $\times 4$ video clips $\times 2$ time points). rMT ranged from 29 to $51 \%$ (mean $=41 \%, \mathrm{SD}=6.1$ ).

\section{Eye tracking recordings}

Eye movements were recorded by means of an infrared Tobii T120 Eye Tracker (Tobii Technology, Danderyd, Sweden), embedded in a 17" display. Eye position was sampled at $120 \mathrm{~Hz}$ with a spatial accuracy of 0.5 deg of visual angle. Prior to starting the experiment, the eye-tracker calibration was performed through a standard five-point grid, and repeated when necessary. Participants were seated at a distance of $65 \mathrm{~cm}$ from the monitor $(1280 \times 1024$ pixels $)$ and they were asked to observe the experimental stimuli (AVI format videos, 25 frames per second). Each trial started with the presentation of a fixation cross in the center of the screen and participants were instructed to look at the cross for $3 \mathrm{~s}$. This ensured that all participants would start observing the video stimuli from the same origin point. Each video clip was presented to each participant three times in a randomized order, for a total of twelve presentations.

\section{Reading the Mind in The Eyes Test (RMET)}

The RMET (Baron-Cohen et al., 2001; Serafin and Surian, 2004) is a well-validated task used to investigate the ability to infer the mental states of others. It involves the presentation of 36 photographs of the eye region of male and female actors, flanked by one correct emotional term out of four. Participants were shown each photograph and asked to choose which word they thought best described what the person in the photograph was thinking or feeling. The total number of correct choices is indicative of the RMET performance. In addition, a gender control task, in which participants had to indicate the gender of the person depicted in the photograph, was administered to control for basic visual discrimination abilities.

\section{Data Analysis}

Data for the RMET and the gender control test were analyzed to verify whether participants scored within the normal range. EMG and statistical analyses were performed as for Experiment 1 , except that three predictors were used as fixed effects of the model - namely Muscle (ADM and FDI), Type of action (Thermos and Spoon) and Gaze direction (Engaged and Averted). Eye-tracking data were analyzed using Tobii studio 3.1 (Tobii Technology, Danderyd, Sweden) to investigate fixations targeted to specific regions of interest (i.e., AOIs; Areas of Interest). A fixation event was computed when gaze remained within 0.5 degrees of visual angle for at least $100 \mathrm{~ms}$. For each video, three AOIs were adopted (see Figure 4): (i) Head AOI $(203 \times 225$ pixels): a static area which included the actor's head; (ii) Hand AOI $(248 \times 228$ pixels): a dynamic area which included the actor's hand while performing the action; and (iii) Object AOI $(129 \times 147$ pixels): a static area covering the mug/cup placed near the observer, in the bottom right corner of the screen. Gaze behavior within the AOIs was measured for the entire duration 

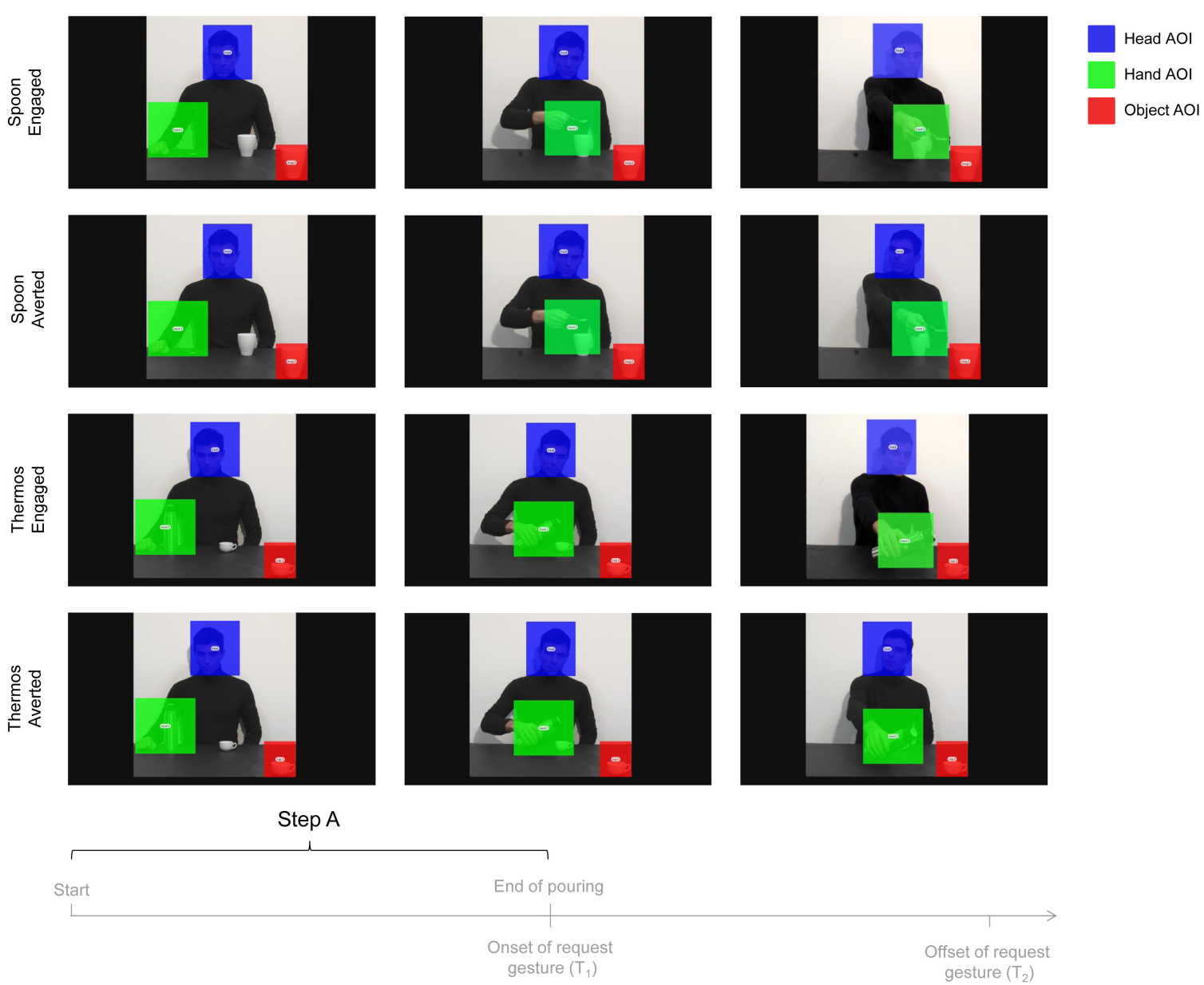

Step B

FIGURE 4 | Sequence of events for the four experimental videos (i.e., Engaged and Averted conditions with Spoon and Thermos actions) and the time epochs considered for data analysis (i.e., Step A and Step B). The overlaid colored rectangular areas represent the adopted AOls: Head AOI (blue); Hand AOI (green); and Object AOI (red).

of the video stimuli. Fixation Duration (i.e., the average duration in seconds for all fixations within the AOI) was specifically investigated during two temporal windows - namely Step A and Step B - preceding and following the start of the social request. The first temporal window included the entire actor's action from movement onset to end of pouring in the close mug/cup (Step A; 6320 ms duration; Figure 4). The second temporal window, instead, comprised the start of the social request to the end of the video clip (Step B; 3360 ms duration; Figure 4). Statistical analyses of the data were performed using a linear mixed-effects model on eye-tracking measures.

\section{Results and Discussion}

All participants scored within the normal range (cutoff $=13 / 36$; see Baron-Cohen et al., 2001) both at the RMET test $(26.67 \pm 2.63)$ and at the RMET gender control test $(35.17 \pm 0.95)$, in line with normative data from an Italian sample of similar age with no previous history of mental disorders $(N=75$, test score: $25 \pm 3.9$, gender control score: $35.4 \pm 0.9$; see Serafin and Surian, 2004). Please refer to Supplementary Table 3 for descriptive statistics of normalized MEP. As concerns the mixed model performed on MEPs considering all the independent variables, the time predictor showed a statistically significant effect $\left(\chi^{2}(1)=17.18, p<0.001\right)$. MEPs at $T_{1}$ (i.e., request gesture onset) were higher than those measured at $\mathrm{T}_{2}$ (i.e., request gesture offset; $t_{(435)}=4.15$, $p<0.0001)$. This might explain the lack of results in Experiment 1, where the TMS pulse was delivered at the request gesture offset. The condition predictor also showed a significant effect $\left(\chi^{2}(1)=8.75, p=0.003\right)$. MEPs for the Engaged conditions were higher than for the Averted conditions $\left(t_{(435)}=-2.96\right.$, $p=0.003)$. Splitting the overall model across the time predictor's levels, at $\mathrm{T}_{1}$ both the condition $\left(\chi^{2}{ }_{(1)}=8, p=0.005\right)$ and the interaction between condition and action $\left(\chi^{2}(1)=5.42\right.$, $p=0.02$ ) were significant. MEPs at $\mathrm{T}_{1}$ for the Engaged conditions were higher than for the Averted conditions $\left(t_{(203)}=-2.82\right.$, 


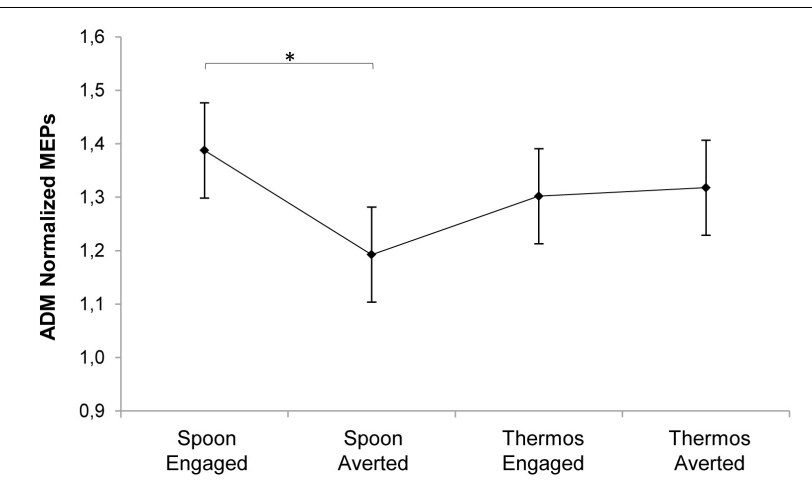

FIGURE 5 | Plot of the interaction between action and condition predictors on MEP normalized amplitude for the ADM muscle at $T_{1}$. Asterisk indicates a statistically significant difference $(p<0.05)$. Bars refer to standard error.

$p=0.005)$. Such a comparison was significant only for the Spoon action $\left(t_{(203)}=-3.65, p=0.002\right)$. Two separate mixed models were performed considering each muscle separately since the adopted intensity of stimulation based on the ADM threshold value may have over-stimulated the FDI muscle. In order to minimize this potential stimulation bias, we conducted the analysis separately for each muscle (see Cavallo et al., 2011 for a similar approach). For the ADM muscle, a statistically significant interaction emerged at $T_{1}$ between action and condition predictors $\left(\chi^{2}(1)=5.3, p=0.02\right)$. MEPs for the Spoon-Engaged condition were higher compared to the Spoon-Averted condition $\left(t_{(87)}=-3, p=0.002\right.$; Figure 5). This indicates that ADM was specifically activated by a request upon the mug (i.e., affording a WHG) when the gaze was pointing to the observer rather than away. For the FDI muscle's model, only a significant effect of the condition predictor emerged at $\mathrm{T}_{1}\left(\chi^{2}{ }_{(1)}=7.89, p=0.005\right)$. MEPs for the Engaged conditions were greater than those for the Averted conditions $\left(t_{(87)}=-2.8, p=0.006\right)$. No significant effect resulted for FDI MEP amplitudes in terms of action predictor, since FDI was involved in the motor preparation of both complementary responses.

\section{Eye-Tracking Results}

Please refer to Supplementary Table 4 for descriptive statistics of fixation duration. As the three AOIs differed in their dimensions, they were considered separately in the analysis for meaningful comparisons. The analysis on the first part of the action (Step A), when the action sequences were identical and no gaze manipulation was presented, led to no statistically significant effects. Conversely, significant effects emerged for all three AOIs (Hand, Head, and Object) during Step B, when the social request was expressed and gaze direction was manipulated (Engaged and Averted). In particular, the Hand AOI presented a significant effect of the condition predictor $\left(\chi^{2}(1)=4.09, p=0.042\right)$. The hand was observed for a longer time in the Engaged compared to the Averted gaze condition $\left(t_{(87)}=-2.02, p=0.046\right)$. After splitting such model across the action predictor's levels, a significant effect was found for the Thermos action $\left(\chi^{2}(1)=6.05\right.$, $p=0.014)$. The hand was observed for a longer time in the
Thermos-Engaged compared to the Thermos-Averted condition $\left(t_{(29)}=-2.46, p=0.02\right)$. As concerns the Head AOI, in the overall model the action predictor presented a statistically significant effect $\left(\chi^{2}(1)=4.02, p=0.045\right)$. The Head was observed for a longer time during the Spoon rather than the Thermos action $\left(t_{(87)}=2, p=0.048\right)$. Finally, considering the Object AOI, the action predictor showed an effect $\left(\chi^{2}(1)=12.04, p<0.001\right)$. The Object located nearby the observer was observed longer during the Thermos action (i.e., while observing a coffee cup) compared to the Spoon action (i.e., while observing a mug; $t_{(87)}=-3.47$, $p<0.001)$.

\section{CONCLUSION}

The aim of the present study was to explore how observed gaze and the implicit request for a complementary action affect motor preparation and gaze behavior in onlookers. In two experiments we specifically investigated the temporal and the spatial aspects of social engagement by manipulating the time and direction of observed gaze and request gestures.

The results from Experiment 1 showed no effect on CE when presenting a request gesture accompanied by a direct gaze to the observer, nor when the actor's gaze was convergent with the direction of his gesture (i.e., both pointing to the target object). In the light of previous results from our laboratory on complementary actions (for reviews, see Sartori and Betti, 2015; Sartori, 2016), this finding was quite unexpected. In those studies we demonstrated corticospinal facilitation while participants observed video-clips evoking complementary gestures. However, it must be said that in all those studies only the actor's arm was shown in the video clips, without the head. Here, we probably hindered the original effect by introducing a source of composite stimuli (i.e., the head and gaze movements) requiring complex processing. Notably, a link emerged between MEPs and readiness to interact. The more participants declared their engagement in the social interaction, the more their CE was activated. This effect was specific for the muscle potentially involved in the complementary response.

Experiment 2 was conceived to deeply investigate social motor preparation, and to disambiguate alternative explanations (see below).

\section{Diverting Attention DOES Affect Complementary Responses}

In terms of $\mathrm{CE}$, the main result of Experiment 2 was an increase of activity in the ADM muscle as soon as the actor's arm started to move the sugar spoon toward the mug placed nearby the observer while looking straight to the her/him, compared to when he was gazing away (i.e., to the contralateral side with respect to the gesture). This finding seems to confirm the hypothesis by Reader and Holmes (2016), suggesting that the separation of gaze and body cues has adverse effects on social interaction.

In a recent TMS experiment with video clips showing similar request gestures but without head and eye cues (Betti et al., 2017), we tested the influence of distracting stimuli on complementary motor preparation. Motor activity in the muscles required to 
perform a complementary response (i.e., a whole-hand grasp) was impervious to the appearance of a dot diverting attention to the contralateral side of the scene with respect to the moving hand, even when it was overtly attended. This suggested that complementary motor preparation is resistant to modulation by top-down mechanisms, such as visuospatial attention. Given the social relevance of understanding the actions of others, it seems plausible that action observation areas in the brain are relatively unaffected by attention modulation. However, data from the present study in which it is the actor's gaze that points to the contralateral side of the scene - instead of a dot - seem to suggest that social motor preparation is highly susceptible to biological cues, able to automatically capture and divert observer's attention. This point to the existence of dedicated mechanisms for special classes of stimuli with particular biological and social significance (e.g., McKone et al., 2007).

\section{Early Social Engagement Enhances Corticospinal Excitability}

The very fact that we found an early corticospinal modulation at $\mathrm{T}_{1}$ (i.e., at the beginning of the social request) but not at $\mathrm{T}_{2}$ (i.e., at the end of the request gesture) highlights two crucial aspects.

First of all, it endorses a previous finding that complementary response preparation promptly occurs and that it is time-locked to the kinematics of the actor's arm movement (i.e., functional shift; Sartori et al., 2013). In that study, video-clips similar to the present stimuli were adopted and TMS was delivered at five different time points corresponding to five kinematic landmarks characterizing the observed action. The most important was an early time point, when the actor's hand trajectory began to move toward the out-of-reach object. A TMS pulse specifically delivered at that time revealed that wrist rotation was the only salient kinematic parameter upon which observers relied to discriminate the intention of the actor, before the request was fully expressed. This confirms previous evidence unveiling the existence of heuristics based on action and gaze cues that allow intention discrimination (Sartori et al., 2011a ; Becchio et al., 2018). When an observed gesture is socially relevant, anticipatory complementary activations follow. The functional shift indicates the ability to untie the automatic tendency to mirror another's actions and to quickly prepare a complementary response.

Secondly, the present finding suggests that the lack of effects shown in Experiment 1 was probably due to a delay in the timing of TMS stimulation. In fact, the single pulse was delivered when the actor's arm was already stretched out toward the target object. This finding is in line with an extensive literature on action anticipation and motor resonance, showing a greater motor facilitation when observing the early stages of an action rather than the action's conclusion (Gangitano et al., 2001, 2004; Aglioti et al., 2008). One suggested function of the motor system is to provide the visual system with predictions about the future state of an unfolding action (Blakemore and Frith, 2005; Wilson and Knoblich, 2005; Prinz, 2006) and this ability allows the selection of a suitable action from a multiplicity of possible alternatives (Bekkering et al., 2009; Sartori et al., 2013). Advance information gained while an action sequence is being observed would allow observers to interact appropriately. These findings have direct implications with regard to action representation theories as they suggest that intention attribution is sensitive to early kinematic cues (Kilner et al., 2007; Sartori et al., 2009, 2011a; Becchio et al., 2010, 2012, 2018; Manera et al., 2011).

A limitation of the current design - and of similar studies is that a single hot spot and a single intensity were chosen for stimulating both the FDI and ADM cortical maps. Notably, the ADM's motor representation is weaker than that of the FDI, since the little finger abduction is a relatively infrequent movement as compared to the index finger abduction that is frequently activated during pointing movements or together with the thumb to grasp and handle objects. It is then possible that setting the rMT based on ADM might have "over-stimulated" FDI (Naish and Obhi, 2015). This hypothesis would be confirmed by the fact that MEPs elicited in FDI were greater than those triggered in ADM. It is therefore possible that differences in stimulation areas, motor representations, and stimulation intensity could have influenced our pattern of results, and this is a factor to bear in mind for future studies.

\section{Gaze Engagement}

Results from Experiment 1 suggest that gazing at the onlooker at the end of the action (i.e., Direct Gaze) increases perceived social engagement more than gazing at the object. Indeed, the more the participants felt involved in the action, the higher was the CE in the muscle required for the interaction. The joint contribution of request gestures and direct gaze maximizes the efficiency of social response preparation. As regards Experiment 2, eye-tracking data confirmed that the engaging gaze was effective in drawing observers' attention both on the actor's hand and on the coffee cup (Thermos condition). No effect was found when the actor's gaze was averted, or when the request gesture was directed to the mug, an object much simpler to be handled than the coffee cup, therefore less complex in terms of affordance. This hypothesis is further confirmed by the fact that participants spent longer time observing the coffee cup than the mug. Lastly, participants observed the actor's head for longer time during the Spoon rather than the Thermos request gesture. It is tempting to assume that the goal of the pouring gesture performed with the spoon was vaguer than the same gesture performed with the thermos. This might have induced participants to spend more time looking the actor's head, in the attempt to clarify his intentions.

Functional imaging studies in adults have shown that activity in a cortical and subcortical network of regions defined as "the social brain network" (Johnson et al., 2005; Adolphs, 2009) specialized in processing social information - is modulated by eye contact (Senju and Johnson, 2009). The present data confirm and extend previous literature suggesting that direct gaze not only signals that a request is relevant for the agent, but it also concurs to quickly activate the appropriate social affordance (Ferri et al., 2011; Innocenti et al., 2012; Letesson et al., 2015). Engaging in complementary interactions, in particular, is made possible by immediate understanding of another person's intentions toward a salient object (Becchio et al., 2018) and the readiness to engage in socially meaningful situations (Di Paolo and De Jaegher, 2012). In this respect, a crucial advantage of adopting dynamic stimuli 
is that they approximate to a real social interaction, and this represents a step forward for the available literature on social interactions. Future studies should capitalize on the finding that social motor preparation increases with early gaze engagement (i.e., direct gaze), followed by a gaze toward the salient object for the interaction; not the other way round.

\section{GENERAL CONCLUSION}

The present research suggests that social motor preparation in interactive contexts is time-locked to the combination of early kinematics cues and direct gaze. This is a novel and interesting finding and it is consistent with recent evidence showing that direct gaze contributes to the prompt activation of appropriate social affordances (Ferri et al., 2011). Complementary affordances, in particular, are a specific subcategory of social affordances referring to all those possibilities for interaction provided by others which activate appropriate motor programs aiming to bring a common goal to completion. Complementary affordances depend on a number of variables, such as: (i) the presence of salient objects necessary for an action to occur, (ii) gaze information, and (iii) the willingness to engage in a collaborative task (Sartori, 2016). The crucial role played by a salient object on the planning of an appropriate response has been widely investigated in past literature. Here, we specifically assessed the role of direct gaze in conjunction with a request gesture on a salient object. Notably, results from Experiment 2 reveal that the separation of body kinematics and averting gaze cues has adverse effects on social interaction. While further research is needed to determine the specific additional role of the third component, namely social engagement, our results are among the first to investigate the role of direct gaze on $\mathrm{CE}$ in social contexts eliciting complementary responses in the onlookers.

\section{REFERENCES}

Adolphs, R. (2009). The social brain: neural basis of social knowledge. Annu. Rev. Psychol. 60, 693-716. doi: 10.1146/annurev.psych.60.110707.163514

Aglioti, S. M., Cesari, P., Romani, M., and Urgesi, C. (2008). Action anticipation and motor resonance in elite basketball players. Nat. Neurosci. 11:1109. doi: $10.1038 / \mathrm{nn} .2182$

Atkinson, M. A., Simpson, A. A., and Cole, G. G. (2017). Visual attention and action: how cueing, direct mapping, and social interactions drive orienting. Psychon. Bull. Rev. doi: 10.3758/s13423-017-1354-0 [Epub ahead of print].

Baron-Cohen, S. (1995a). Mind Blindness: An Essay on Autism and Theory of Mind. Cambridge, MA: MIT Press.

Baron-Cohen, S. (1995b). "The eye direction detector (EDD) and the shared attention mechanism (SAM): two cases for evolutionary psychology," in Joint Attention: Its Origins and Role in Development, eds C. Moore and P. J. Dunham (Hillsdale: Lawrence Erlbaum Associates), 41-59.

Baron-Cohen, S., Wheelwright, S., Hill, J., Raste, Y., and Plumb, I. (2001). The "reading the mind in the eyes" test revised version: a study with normal adults, and adults with asperger syndrome or high-functioning autism. J. Child Psychol. Psychiatry 42, 241-251. doi: 10.1017/S0021963001006643

Basil-Neto, J. P., Cohen, L. G., Panizza, M., Nilsson, J., Roth, B. J., and Hallett, M. (1992). Optimal focal transcranial magnetic activation of the human motor
The data outlined here might contribute to shedding light on the functioning of the human motor system in social contexts and to increase our knowledge on forms of social disorders, such as autism. In computational terms, one of the long-term goals of the present and other studies (Chinellato et al., 2013) is to implement neurophysiological data into a model able to provide artificial systems, such as humanoid robots, with more advanced social skills as they interact with humans.

\section{AUTHOR CONTRIBUTIONS}

SB, UG, and LS designed the study and analyzed the data. GZ and SG created the stimuli and collected the data. SB, UG, UC, and LS interpreted the data and discussed the results. SB and LS wrote the manuscript. SB, UC, and LS critically revised the manuscript.

\section{FUNDING}

This work was supported by Progetto Strategico, Università di Padova (N. 2010XPMFW4) to UC and by SIR grant (Scientific Independence of Young Researchers - N. RBSI141QKX) to LS.

\section{ACKNOWLEDGMENTS}

The authors would like to thank Nicolò Granata and Stefano Santacroce for their help in conducting Experiment 1 and Alice Groselli for her help in conducting Experiment 2.

\section{SUPPLEMENTARY MATERIAL}

The Supplementary Material for this article can be found online at: https://www.frontiersin.org/articles/10.3389/fpsyg. 2018.01408/full\#supplementary-material

cortex: effects of coil orientation, shape of the induced current pulse, and stimulus intensity. J. Clin. Neurophysiol. 9, 132-136. doi: 10.1097/00004691199201000-00014

Bates, D., Mächler, M., Bolker, B., and Walker, S. (2014). Fitting linear mixed-effects models using lme4. ArXiv14065823 Stat. Available at: http://arxiv.org/abs/1406. 5823

Batki, A., Baron-Cohen, S., Wheelwright, S., Connellan, J., and Ahluwalia, J. (2000). Is there an innate gaze module? Evidence from human neonates. Infant Behav. Dev. 23, 223-229. doi: 10.1016/S0163-6383(01)00037-6

Becchio, C., Koul, A., Ansuini, C., Bertone, C., and Cavallo, A. (2018). Seeing mental states: an experimental strategy for measuring the observability of other minds. Phys. Life Rev. 24, 67-80. doi: 10.1016/j.plrev.2017.10.002

Becchio, C., Manera, V., Sartori, L., Cavallo, A., and Castiello, U. (2012). Grasping intentions: from thought experiments to empirical evidence. Front. Hum. Neurosci. 6:117. doi: 10.3389/fnhum.2012.00117

Becchio, C., Sartori, L., and Castiello, U. (2010). Toward you: the social side of actions. Curr. Dir. Psychol. Sci. 19, 183-188. doi: 10.1177/0963721410 370131

Bekkering, H., de Bruijn, E. R. A., Cuijpers, R. H., Newman-Norlund, R., Van Schie, H. T., and Meulenbroek, R. (2009). Joint action: neurocognitive mechanisms supporting human interaction. Top. Cogn. Sci. 1, 340-352. doi: 10.1111/j.17568765.2009.01023.x 
Betti, S., Castiello, U., Guerra, S., and Sartori, L. (2017). Overt orienting of spatial attention and corticospinal excitability during action observation are unrelated. PLoS One 12:e0173114. doi: 10.1371/journal.pone. 0173114

Betti, S., Zani, G., Guerra, S., Castiello, U., and Sartori, L. (2018). Reach-to-grasp movements: a multimodal techniques study. Front. Psychol. 9:990. doi: 10.3389/ fpsyg.2018.00990

Blakemore, S. -J., and Frith, C. (2005). The role of motor contagion in the prediction of action. Neuropsychologia 43, 260-267. doi: 10.1016/j. neuropsychologia.2004.11.012

Castiello, U. (2003). Understanding other people's actions: intention and attention. J. Exp. Psychol. Hum. Percept. Perform. 29, 416-430. doi: 10.1037/0096-1523.29. 2.416

Cavallo, A., Sartori, L., and Castiello, U. (2011). Corticospinal excitability modulation to hand muscles during the observation of appropriate versus inappropriate actions. Cogn. Neurosci. 2, 83-90. doi: 10.1080/17588928.2010. 533163

Chinellato, E., Ognibene, D., Sartori, L., and Demiris, Y. (2013). Time to Change: Deciding When to Switch Action Plans During a Social Interaction. in Biomimetic and Biohybrid Systems Lecture Notes in Computer Science. Berlin: Springer, 47-58. doi: 10.1007/978-3-642-39802-5_5

Classen, J., Steinfelder, B., Liepert, J., Stefan, K., Celnik, P., Cohen, L. G., et al. (2000). Cutaneomotor integration in humans is somatotopically organized at various levels of the nervous system and is task dependent. Exp. Brain Res. 130, 48-59. doi: 10.1007/s002210050005

Di Paolo, E., and De Jaegher, H. (2012). The interactive brain hypothesis. Front. Hum. Neurosci. 6:163. doi: 10.3389/fnhum.2012.00163

Driver, J. I., Davis, G., Ricciardelli, P., Kidd, P., Maxwell, E., and Baron-Cohen, S. (1999). Gaze Perception triggers reflexive visuospatial orienting. Vis. Cogn. 6, 509-540. doi: 10.1080/135062899394920

Emery, N. J. (2000). The eyes have it: the neuroethology, function and evolution of social gaze. Neurosci. Biobehav. Rev. 24, 581-604. doi: 10.1016/S0149-7634(00) 00025-7

Farroni, T., Csibra, G., Simion, F., and Johnson, M. H. (2002). Eye contact detection in humans from birth. Proc. Natl. Acad. Sci. U.S.A. 99, 9602-9605. doi: 10.1073/ pnas. 152159999

Ferri, F., Campione, G. C., Volta, R. D., Gianelli, C., and Gentilucci, M. (2011). Social requests and social affordances: how they affect the kinematics of motor sequences during interactions between conspecifics. PLoS One 6:e15855. doi: 10.1371/journal.pone.0015855

Fox, J., and Weisberg, S. (2011). An R Companion to Applied Regression, 2nd Edn. Thousand Oaks, CA: Sage.

Friesen, C. K., Moore, C., and Kingstone, A. (2005). Does gaze direction really trigger a reflexive shift of spatial attention? Brain Cogn. 57, 66-69. doi: 10.1016/ j.bandc.2004.08.025

Frischen, A., Bayliss, A. P., and Tipper, S. P. (2007). Gaze cueing of attention: visual attention, social cognition, and individual differences. Psychol. Bull. 133, 694-724. doi: 10.1037/0033-2909.133.4.694

Gangitano, M., Mottaghy, F. M., and Pascual-Leone, A. (2001). Phasespecific modulation of cortical motor output during movement observation. Neuroreport 12, 1489-1492. doi: 10.1097/00001756-200105250-00038

Gangitano, M., Mottaghy, F. M., and Pascual-Leone, A. (2004). Modulation of premotor mirror neuron activity during observation of unpredictable grasping movements. Eur. J. Neurosci. 20, 2193-2202. doi: 10.1111/j.1460-9568.2004. 03655.x

Gaylor, D. W., and Hopper, F. N. (1969). Estimating the degrees of freedom for linear combinations of mean squares by satterthwaite's formula. Technometrics 11, 691-706. doi: 10.1080/00401706.1969.10490732

George, N., and Conty, L. (2008). Facing the gaze of others. Neurophysiol. Clin. Neurophysiol. 38, 197-207. doi: 10.1016/j.neucli.2008.03.001

Hamilton, A. F. C. (2016). Gazing at me: the importance of social meaning in understanding direct-gaze cues. Philos. Trans. R Soc. B 371:20150080. doi: $10.1098 /$ rstb. 2015.0080

Innocenti, A., Stefani, E. D., Bernardi, N. F., Campione, G. C., and Gentilucci, M. (2012). Gaze Direction and request gesture in social interactions. PLoS One 7:e36390. doi: 10.1371/journal.pone.0036390

Johnson, M. H., Griffin, R., Csibra, G., Halit, H., Farroni, T., Haan, M. D., et al. (2005). The emergence of the social brain network: evidence from typical and atypical development. Dev. Psychopathol. 17, 599-619. doi: 10.1017/ S0954579405050297

Kilner, J. M., Friston, K. J., and Frith, C. D. (2007). Predictive coding: an account of the mirror neuron system. Cogn. Process. 8, 159-166. doi: 10.1007/s10339-0070170-2

Kleinke, C. L. (1986). Gaze and eye contact: a research review. Psychol. Bull. 100, 78-100. doi: 10.1037/0033-2909.100.1.78

Kobayashi, H., and Kohshima, S. (2001). Unique morphology of the human eye and its adaptive meaning: comparative studies on external morphology of the primate eye. J. Hum. Evol. 40, 419-435. doi: 10.1006/jhev.2001.0468

Langton, S. R. H., and Bruce, V. (1999). Reflexive visual orienting in response to the social attention of others. Vis. Cogn. 6, 541-567. doi: 10.1080/135062899394939

Langton, S. R. H., Watt, R. J., and Bruce, V. (2000). Do the eyes have it? Cues to the direction of social attention. Trends Cogn. Sci. 4, 50-59. doi: 10.1016/S13646613(99)01436-9

Lenth, R. V. (2016). Least-squares means: the r package lsmeans. J. Stat. Softw. 69, 1-33. doi: 10.18637/jss.v069.i01

Letesson, C., Grade, S., and Edwards, M. G. (2015). Different but complementary roles of action and gaze in action observation priming: insights from eye- and motion-tracking measures. Front. Psychol. 6:569. doi: 10.3389/fpsyg.2015.00569

Manera, V., Becchio, C., Cavallo, A., Sartori, L., and Castiello, U. (2011). Cooperation or competition? Discriminating between social intentions by observing prehensile movements. Exp. Brain Res. 211, 547-556. doi: 10.1007/ s00221-011-2649-4

McKone, E., Kanwisher, N., and Duchaine, B. C. (2007). Can generic expertise explain special processing for faces? Trends Cogn. Sci. 11, 8-15. doi: 10.1016/ j.tics.2006.11.002

Mills, K. R., Boniface, S. J., and Schubert, M. (1992). Magnetic brain stimulation with a double coil: the importance of coil orientation. Electroencephalogr. Clin. Neurophysiol. Potentials Sect. 85, 17-21. doi: 10.1016/0168-5597(92)90 096-T

Naish, K. R., and Obhi, S. S. (2015). Timing and specificity of early changes in motor excitability during movement observation. Exp. Brain Res. 233, 1867-1874. doi: 10.1007/s00221-015-4258-0

Oldfield, R. C. (1971). The assessment and analysis of handedness: the edinburgh inventory. Neuropsychologia 9, 97-113. doi: 10.1016/0028-3932(71)90067-4

Pierno, A. C., Becchio, C., Wall, M. B., Smith, A. T., Turella, L., and Castiello, U. (2006). When gaze turns into grasp. J. Cogn. Neurosci. 18, 2130-2137. doi: 10.1162/jocn.2006.18.12.2130

Prinsen, J., Bernaerts, S., Wang, Y., de Beukelaar, T. T., Cuypers, K., Swinnen, S. P., et al. (2017). Direct eye contact enhances mirroring of others' movements: a transcranial magnetic stimulation study. Neuropsychologia 95, 111-118. doi: 10.1016/j.neuropsychologia.2016.12.011

Prinz, W. (2006). What re-enactment earns us. Cortex 42, 515-517. doi: 10.1016/ S0010-9452(08)70389-7

R Core Team (2016). R: A Language and Environment for Statistical Computing. Vienna: R Foundation for Statistical Computing. Available at: https://www.Rproject.org/

Reader, A. T., and Holmes, N. P. (2016). Examining ecological validity in social interaction: problems of visual fidelity, gaze, and social potential. Cult. Brain 4 , 134-146. doi: 10.1007/s40167-016-0041-8

Rossi, S., Hallett, M., Rossini, P. M., and Pascual-Leone, A. (2009). Safety, ethical considerations, and application guidelines for the use of transcranial magnetic stimulation in clinical practice and research. Clin. Neurophysiol. 120, 20082039. doi: 10.1016/j.clinph.2009.08.016

Rossini, P. M., Barker, A. T., Berardelli, A., Caramia, M. D., Caruso, G., Cracco, R. Q., et al. (1994). Non-invasive electrical and magnetic stimulation of the brain, spinal cord and roots: basic principles and procedures for routine clinical application. Report of an IFCN committee. Electroencephalogr. Clin. Neurophysiol. 91, 79-92. doi: 10.1016/0013-4694(94)90029-9

Sartori, L. (2016). “Complementary actions," in Shared Representations, eds S. S. Obhi and E. S. Cross (Cambridge: Cambridge University Press), 392-416. doi: 10.1017/CBO9781107279353.020

Sartori, L., and Betti, S. (2015). Complementary actions. Front. Psychol. 6:557. doi: $10.3389 /$ fpsyg.2015.00557

Sartori, L., Becchio, C., and Castiello, U. (2011a). Cues to intention: the role of movement information. Cognition 119, 242-252. doi: 10.1016/j.cognition.2011. 01.014 
Sartori, L., Becchio, C., Bulgheroni, M., and Castiello, U. (2009). Modulation of the action control system by social intention: unexpected social requests override preplanned action. J. Exp. Psychol. Hum. Percept. Perform. 35, 1490-1500. doi: $10.1037 / \mathrm{a} 0015777$

Sartori, L., Bucchioni, G., and Castiello, U. (2013). When emulation becomes reciprocity. Soc. Cogn. Affect. Neurosci. 8, 662-669. doi: 10.1093/scan/nss044

Sartori, L., Cavallo, A., Bucchioni, G., and Castiello, U. (2011b). Corticospinal excitability is specifically modulated by the social dimension of observed actions. Exp. Brain Res. 211:557. doi: 10.1007/s00221-0112650-y

Schilbach, L., Timmermans, B., Reddy, V., Costall, A., Bente, G., Schlicht, T., et al. (2013). Toward a second-person neuroscience. Behav. Brain Sci. 36, 393-462. doi: 10.1017/S0140525X12000660

Sebanz, N., Bekkering, H., and Knoblich, G. (2006). Joint action: bodies and minds moving together. Trends Cogn. Sci. 10, 70-76. doi: 10.1016/j.tics.2005.12.009

Senju, A., and Johnson, M. H. (2009). The eye contact effect: mechanisms and development. Trends Cogn. Sci. 13, 127-134. doi: 10.1016/j.tics.2008.11.009

Serafin, M., and Surian, L. (2004). Il Test degli Occhi: uno strumento per valutare la "teoria della mente." G. Ital. Psicol. 4:839-862. doi: 10.1421/1 8849
Wassermann, E. M. (1998). Risk and safety of repetitive transcranial magnetic stimulation: report and suggested guidelines from the International Workshop on the Safety of Repetitive Transcranial Magnetic Stimulation, June 5-7, 1996. Electroencephalogr. Clin. Neurophysiol. Potentials Sect. 108, 1-16. doi: 10.1016/ S0168-5597(97)00096-8

Wilson, M., and Knoblich, G. (2005). The case for motor involvement in perceiving conspecifics. Psychol. Bull. 131, 460-473. doi: 10.1037/0033-2909.131. 3.460

Conflict of Interest Statement: The authors declare that the research was conducted in the absence of any commercial or financial relationships that could be construed as a potential conflict of interest.

Copyright (C) 2018 Betti, Zani, Granziol, Guerra, Castiello and Sartori. This is an open-access article distributed under the terms of the Creative Commons Attribution License (CC BY). The use, distribution or reproduction in other forums is permitted, provided the original author(s) and the copyright owner(s) are credited and that the original publication in this journal is cited, in accordance with accepted academic practice. No use, distribution or reproduction is permitted which does not comply with these terms. 